\title{
Global Properties in High Energy Collisions
}

\author{
Bao-Chun Li, ${ }^{1}$ Edward Sarkisyan-Grinbaum, ${ }^{2,3}$ Andrey Leonidov, ${ }^{4}$ \\ Venktesh Singh, ${ }^{5}$ and $\mathrm{C}$. $\mathrm{Wu}^{6}$ \\ ${ }^{1}$ Department of Physics, Shanxi University, Taiyuan, Shanxi 030006, China \\ ${ }^{2}$ PH Department, CERN, 1211 Geneva 23, Switzerland \\ ${ }^{3}$ Department of Physics, The University of Texas at Arlington, Arlington, TX 76019, USA \\ ${ }^{4}$ Theoretical Physics Department, P.N. Lebedev Physics Institute, Moscow 119991, Russia \\ ${ }^{5}$ Department of Physics, Banaras Hindu University, Varanasi 221005, India \\ ${ }^{6}$ Shanghai Institute of Applied Physics, Chinese Academy of Sciences, Shanghai 204001, China \\ Correspondence should be addressed to Bao-Chun Li; libc2010@163.com
}

Received 4 March 2015; Accepted 4 March 2015

Copyright (C) 2015 Bao-Chun Li et al. This is an open access article distributed under the Creative Commons Attribution License, which permits unrestricted use, distribution, and reproduction in any medium, provided the original work is properly cited. The publication of this article was funded by $\mathrm{SCOAP}^{3}$.

The nuclear and particle collisions at high energy provide a unique environment to explore different kinds of phase transitions related to the formation of the quark matter and the confinement phenomena. Global properties of particles produced in the collisions are crucial information on the main features of the evolution of the initially created dense quark system into the stream of final hadrons. In highenergy collisions, the investigations help to understand the properties of strongly coupled quark-gluon plasma (sQGP) by testing various production mechanisms.

Thermal-statistical models and many phenomenological models of initial coherent multiple interactions and particle transport have been proposed to discuss the global properties, such as anisotropy flows, jet properties, multiplicity spectrum, and distributions of different kinematic variables. Due to the complexity of QCD in the regime of strong coupling, results on hot quark matter from lattice calculation and hydrodynamic simulation are still lacking the analytic understanding. Further theoretical investigations are of high interest to explain new features observed in high-energy collisions at LHC and to make predictions for future measurements.

The present issue will publish 13 original research papers or review papers, which cover many important topics in global properties of produced particles in high-energy collisions, such as transverse momentum spectra of hadrons produced in $\mathrm{Au}-\mathrm{Au}, \mathrm{Pb}-\mathrm{Pb}, \mathrm{d}-\mathrm{Au}$, and $p-p$ collisions at RHIC and LHC energies, transverse momentum spectra of protons and charged pions in $p-\mathrm{Cu}$ and $p-\mathrm{Pb}$ collisions measured in Hadron Production Experiment (HARP) at the CERN, dihadron azimuthal correlations in $\mathrm{Au}-\mathrm{Au}, \mathrm{Pb}-\mathrm{Pb}$, and $\mathrm{p}$ $\mathrm{Pb}$ collisions at RHIC and LHC energies, multiplicity and transverse energy production at high energy, elliptic flows in $\mathrm{Pb}-\mathrm{Au}$ collisions at SPS energies, long-range correlations in ${ }^{16} \mathrm{O}-\mathrm{AgBr}$ and ${ }^{32} \mathrm{~S}-\mathrm{AgBr}$ at SPS energies, and multiplicity distributions in the interactions of different nuclei with emulsion (EM) at high energy.

Transverse momentum of the final-state particles is called first observations in the high-energy experiments. To describe such many-particle system, statistical approaches have been used widely over past few years. Transverse momentum spectra in high-energy collisions have been discussed from different perspectives in the papers of B.C. Li et al. "Tsallis Statistical Interpretation of Transverse Momentum Spectra in High-Energy pA Collisions"; J. H. Kang et al. "Emission of Protons and Charged Pions in $\mathrm{p}+$ $\mathrm{Cu}$ and $\mathrm{p}+\mathrm{Pb}$ Collisions at 3, 8, and $15 \mathrm{GeV} / \mathrm{c}$ "; H.-R. Wei et al. "Comparing Multicomponent Erlang Distribution 
and Lévy Distribution of Particle Transverse Momentums"; L.-L. Wang "Transverse Momentum Distributions in $\mathrm{AuAu}$ and dAu Collisions at $\sqrt{s_{N N}}=200 \mathrm{GeV}$ "; F.-H. Liu et al. "On Descriptions of Particle Transverse Momentum Spectra in High Energy Collisions"; S. Uddin et al. "Transverse Momentum Distributions of Hadrons Produced in $\mathrm{Pb}-\mathrm{Pb}$ Collisions at LHC Energy $\sqrt{s_{N N}}=2.76 \mathrm{TeV}$ "; and $\mathrm{H}$. Zhao and F.-H. Liu "On Extraction of Chemical Potentials of Quarks from Particle Transverse Momentum Spectra in High Energy Collisions."

In the framework of a multisource thermal model, the papers of F.-H. Liu et al. "On Two-Ridge Structure in TwoParticle Azimuthal Correlations in Proton-lead Collisions at Large Hadron Collider Energy" and G. X. Zhang et al. "Dihadron Azimuthal Correlations in $200 \mathrm{GeV} \mathrm{Au}-\mathrm{Au}$ and 2.76 TeV Pb-Pb Collisions" study in detail dihadron azimuthal correlations for the different centralities in $\mathrm{Au}-\mathrm{Au}$ collisions at $\sqrt{s_{N N}}=200 \mathrm{GeV}$, in $\mathrm{Pb}-\mathrm{Pb}$ collisions at $\sqrt{s_{N N}}=$ $2.76 \mathrm{TeV}$, and in $p-\mathrm{Pb}$ at $\sqrt{s_{N N}}=5.02 \mathrm{TeV}$. Each particle pair measured in final state is considered to be produced from a single or several sources. The model can approximately describe the azimuthal correlations of particles produced in the collisions. In the model, the paper of S. Fakhraddin et al. "Shower and Slow Particle Productions in NucleusNucleus Collisions at High Energy" discusses the multiplicity distributions of shower, grey, and black particles produced in interactions of ${ }^{4} \mathrm{He},{ }^{12} \mathrm{C},{ }^{16} \mathrm{O},{ }^{22} \mathrm{Ne}$, and ${ }^{28} \mathrm{Si}$ with emulsion (Em) at 4.1-4.5 AGeV/c beam energies and their dependence on target groups ( $\mathrm{H}, \mathrm{CNO}$, and $\mathrm{AgBr}$ ).

At SPS energies, there are two papers published. The paper of S. Ahmad et al. "Search for Long-Range Correlations in Relativistic Heavy-Ion Collisions at SPS Energies" searches for long-range correlations by analysing the experimental data on ${ }^{16} \mathrm{O}-\mathrm{AgBr}$ and ${ }^{32} \mathrm{~S}-\mathrm{AgBr}$ collisions at $200 \mathrm{AGeV} / \mathrm{c}$ and the results are compared with the predictions of $\mathrm{A}$ Multi Phase Transport (AMPT) model. The paper of B.-C. Li and Z. Zhang "On Particle Production in Lead-Gold Collision and Azimuthal Anisotropy at Top SPS Energy" analyzes the dependence of elliptic flow $v_{2}$ on the transverse momentum $P_{T}$. The model results are compared with the data in $\mathrm{Pb}-\mathrm{Au}$ collisions at top SPS energy, $17.3 \mathrm{GeV}$.

In the review paper of R. Sahoo et al. "Charged Particle, Photon Multiplicity, and Transverse Energy Production in High-Energy Heavy-Ion Collisions," the authors review the charged particle, photon multiplicities, and transverse energy production in heavy-ion collisions starting from few $\mathrm{GeV}$ to $\mathrm{TeV}$ energies. They also discuss the hypothesis of limiting fragmentation and expansion dynamics using the Landau hydrodynamics and the underlying physics. This review overall provides a scope to understand the heavy-ion collision data and a possible formation of a deconfined phase of partons via the global observables like charged particles, photons, and the transverse energy measurement.

Although many topics are touched upon, it is not enough to cover the entire field of global properties in high-energy collisions. We hope that the topic could be continued to track the updated trends year by year. The continuous further progress in high-energy collisions will be helpful for us to understand the matter production.

\section{Acknowledgments}

We sincerely thank all the contributors of this special issue for their support and sincerely thank all the qualified reviewers.

Bao-Chun Li Edward Sarkisyan-Grinbaum Andrey Leonidov Venktesh Singh C. $W u$ 

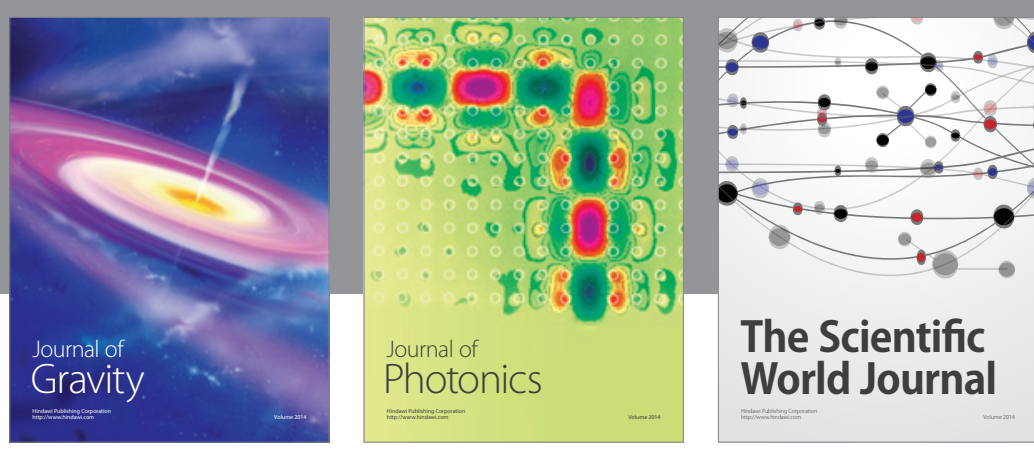

The Scientific World Journal
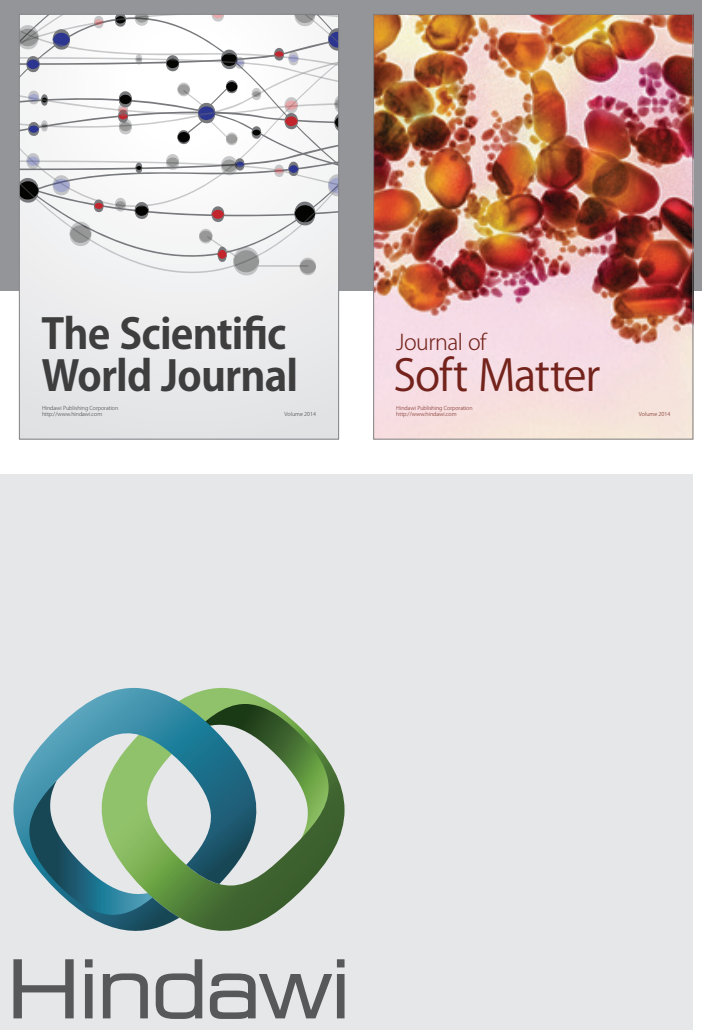

Submit your manuscripts at

http://www.hindawi.com

nternational Journal of

Statistical Mechanics
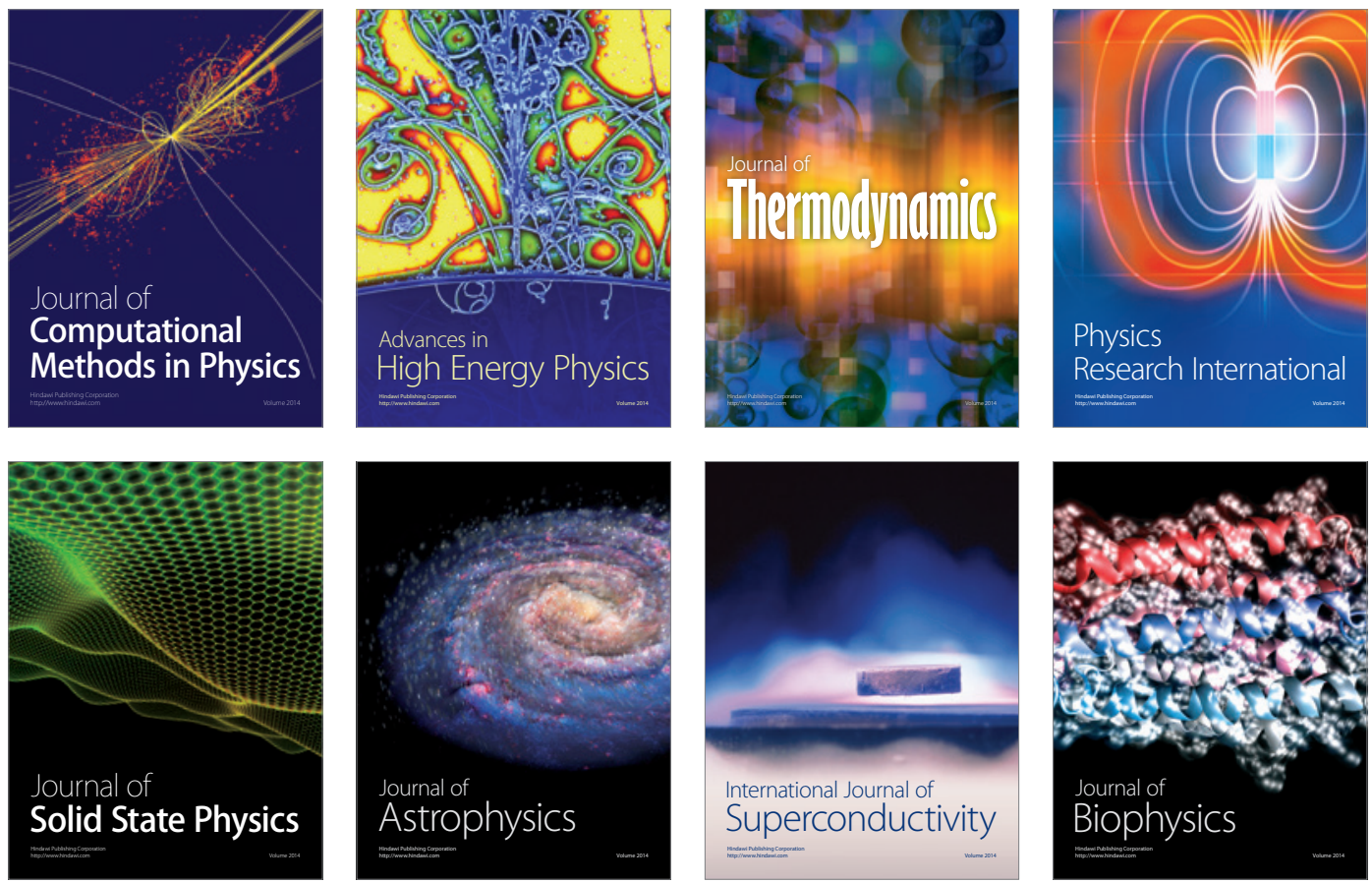
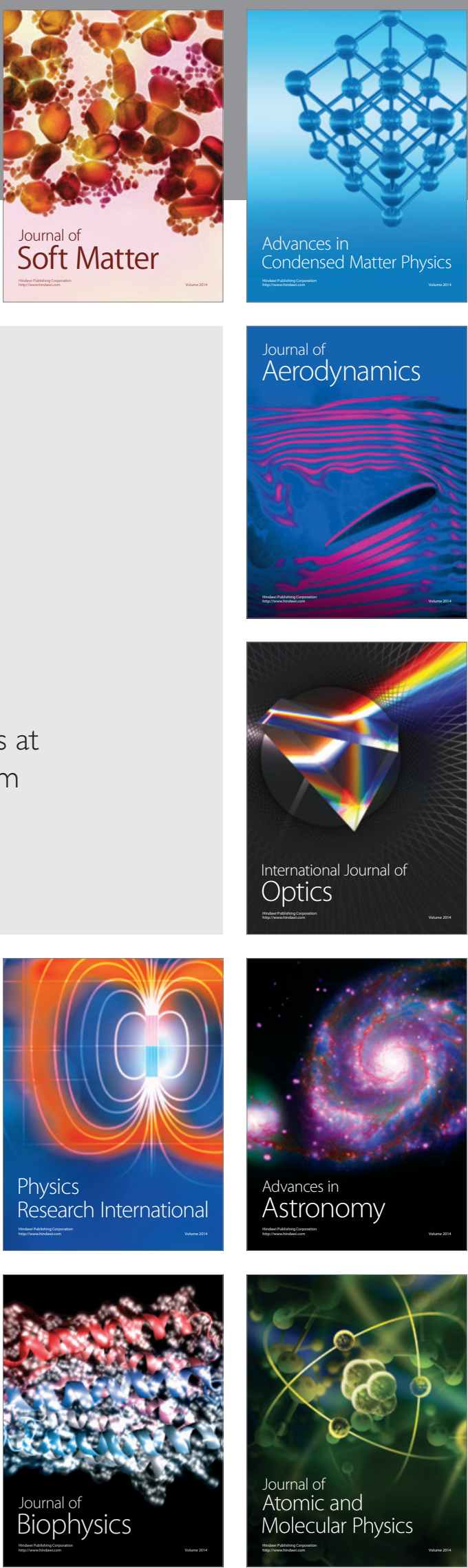